\title{
Simultaneously enhanced strength and ductility for 3D-printed stainless steel 316L by selective laser melting
}

\author{
Zhongji Sun ${ }^{1}$, Xipeng Tan $\mathbb{D}^{1}$, Shu Beng Tor ${ }^{1}$ and Chee Kai Chua ${ }^{1}$
}

\begin{abstract}
Laser-based powder-bed fusion additive manufacturing or three-dimensional printing technology has gained tremendous attention due to its controllable, digital, and automated manufacturing process, which can afford a refined microstructure and superior strength. However, it is a major challenge to additively manufacture metal parts with satisfactory ductility and toughness. Here we report a novel selective laser melting process to simultaneously enhance the strength and ductility of stainless steel $316 \mathrm{~L}$ by in-process engineering its microstructure into a $<011>$ crystallographic texture. We find that the tensile strength and ductility of SLM-built stainless steel $316 \mathrm{~L}$ samples could be enhanced by $\sim 16 \%$ and $\sim 40 \%$ respectively, with the engineered $<011>$ textured microstructure compared to the common <001> textured microstructure. This is because the favorable nano-twinning mechanism was significantly more activated in the $<011>$ textured stainless steel $316 \mathrm{~L}$ samples during plastic deformation. In addition, kinetic simulations were performed to unveil the relationship between the melt pool geometry and crystallographic texture. The new additive manufacturing strategy of engineering the crystallographic texture can be applied to other metals and alloys with twinning-induced plasticity. This work paves the way to additively manufacture metal parts with high strength and high ductility.
\end{abstract}

\section{Introduction}

Metal additive manufacturing (AM), also known as metal three-dimensional (3D) printing, can produce highstrength complex geometries ${ }^{1}$ to obtain components with fine microstructures ${ }^{2}$ and achieve microstructural control within parts ${ }^{3}$. However, metal AM builds typically suffer from poor mechanical properties, such as low ductility and inconsistency, due to the presence of lack-of-fusion defects, internal pores, residual stresses, and anisotropy ${ }^{4,5}$. Post-processing treatments are generally required to restore the desired toughness ${ }^{6}$. Selective laser melting (SLM) has been become a mainstream powder-bed fusion metal AM technique due to its refined resolution and widespread adoption ${ }^{7}$. It is commonly accepted that SLM-

\footnotetext{
Correspondence: Xipeng Tan (xptan1985@gmail.com) (xptan@ntu.edu.sg) ${ }^{1}$ Singapore Centre for 3D Printing, School of Mechanical \& Aerospace Engineering, Nanyang Technological University, 50 Nanyang Avenue, Singapore 639798, Singapore

These authors contributed equally: Zhongji Sun, Xipeng Tan.
}

built parts experience a layer-wise cyclic thermal history ${ }^{8}$. Highly dense parts $(>99 \%)$ can be realized by optimizing the laser scanning parameters ${ }^{9}$. They normally possess a strong $<001>$ crystallographic texture along the build direction against the heat dissipation route ${ }^{10}$. Epitaxial growth is frequently observed ${ }^{11}$ and is hypothesized to be a prerequisite for the formation of various crystallographic textures $^{12}$. Owing to the rapid cooling $\left(\sim 10^{6} \mathrm{~K} / \mathrm{s}\right)$ to ambient temperature involved in the SLM process ${ }^{11}$, elemental segregation is greatly suppressed, while large quantities of martensitic phases and dislocations are favored ${ }^{13}$. Therefore, SLM-built components tend to have higher strength than their counterparts processed by other metal AM techniques. However, the decrease in ductility accompanied with reduced toughness remains to be an unresolved issue in metal AM, especially for the SLM process.

Inspired by the various crystallographic textures ${ }^{11,14,15}$ and grain morphologies ${ }^{16}$ achieved via different scanning strategies in SLM, microstructural control within the 
SLM-built part holds the key to property enhancement. In addition to the common $<001>$ crystallographic texture obtained through SLM, a few studies also attained $<011>$ crystallographic texture along the build direction ${ }^{3,11,14,17}$. Moreover, nanometer-scale twinning (nano-twinning) is shown to be of utmost importance for ductility improvement in the latest research on metals and alloys ${ }^{18-20}$. Unlike conventional grain boundaries or interphase interfaces, the twin boundary energy is low, and it possesses a greater dislocation storage capacity ${ }^{21}$. Dislocations can pass through or glide along the twin boundaries by dissolving into partial dislocations ${ }^{21}$, which could simultaneously enhance both the strength and the ductility of a material.

In this work, we aim to overcome the trade-off between strength and ductility in SLM-built metals and alloys by the in situ tailoring of specific crystallographic textures and grain sizes. Stainless steel 316L (SS316L) is chosen as a representative material for this purpose because it is a popular material employed in metal AM with broad applications ${ }^{10,22,23}$. Moreover, SS316L is a face-centered cubic (FCC) metal with a relatively low stacking fault energy of $\sim 20 \mathrm{~mJ} / \mathrm{m}^{2}$, which favors deformation-induced twinning $^{24}$. This work shows that, by utilizing a specific laser scanning strategy, a favorable fine-grained microstructure with a $<011>$ crystallographic texture could be produced. This aids the activation of the nano-twinning mechanism during the tensile deformation of SLM-built SS316L at room temperature, which simultaneously increases the strength and ductility. This work proposes a promising solution to improve the mechanical performance of metal AM components while using less material.

\section{Materials and methods}

\section{Sample preparation}

All samples were fabricated with an SLM $250 \mathrm{HL}$ machine (SLM Solutions Group AG, Lübeck, Germany). The precursor material was spherical gas-atomized SS316L powder with a size range of $20-63 \mu \mathrm{m}$. A dual laser system was employed, where the laser has a Gaussian profile for power inputs below $400 \mathrm{~W}$ and a top-hat profile for inputs up to 1 $\mathrm{kW}$. The samples to test the process parameters were built with dimensions of $10 \mathrm{~mm}^{3}$, and at least two samples per parameter set were built. Standard mechanical polishing procedures were adopted and can be found elsewhere ${ }^{10}$. Both chemical ( $\sim 30 \mathrm{~min})$ and electrolytic $(\sim 10 \mathrm{~s})$ etchings were employed with Kroll's reagent (1-3\% $\mathrm{HF}, 2-6 \% \mathrm{HNO}_{3}$, and $91-97 \% \mathrm{H}_{2} \mathrm{O}$ ) before optical microscopic $(\mathrm{OM})$ and scanning electron microscopic (SEM) examination.

\section{X-ray diffraction (XRD) and mechanical testing}

XRD analysis was conducted on a PANalytical Empyrean with $\mathrm{Cu} \mathrm{K \alpha}$ radiation. A step size of $0.01^{\circ}$ was used for all samples. The tensile coupon blocks were built with dimensions of $14 \times 36 \times 10 \mathrm{~mm}^{3}$. They were then machined to the tensile coupon size (see Supplementary Fig. 1(d)) via the electrical discharge machining method. The samples were ground with \#320 grit sand paper followed by \#1000 grit. Tensile tests were performed on an Instron static tester (series 5569) with a strain rate of $2.65 \times 10^{-4} / \mathrm{s}$. A contact-based extensometer was used until break. At least three samples for each set were tested.

\section{Monte Carlo simulations}

Kinetic Monte Carlo simulations were conducted with the open-source Stochastic Parallel PARticle Kinetic Simulator ${ }^{25,26}$. The Monte Carlo Potts model that involves spins (or grain identifies) on a discrete lattice was employed. The overall bond energies between adjoining unlike spins (adjacent grains) were computed as $E=\frac{1}{2} \sum_{i=1}^{N} \sum_{j=1}^{L}\left(1-\delta\left(q_{i} q_{j}\right)\right)$, where $N$ is the total number of lattice sites, $q_{i}$ and $q_{j}$ are the spins at lattices sites $i$ and $j$, and $L$ is the number of neighbors of each lattice site. $A$ is the mobility factor and $F$ is specified as $F=F_{o} \exp \left(\frac{-Q}{k_{B} T(t, i)}\right)$, where $Q$ is the activation energy for grain boundary motion, $F_{\mathrm{o}}$ is a user defined prefatory, $K_{\mathrm{B}}$ is Boltzmann's constant, and $T(t, i)$ is the temperature input at a specific time and location. The Monte Carlo acceptance probability of spin flip was then calculated as $P=\left\{\begin{array}{cc}F \exp \left(\frac{-\Delta E}{k_{B} T_{s}}\right) & \text { if } \Delta E>0 \\ F & \text { if } \Delta E \leq 0\end{array}\right.$. This ensures that the overall energy of the system decreases with each calculation step and thus grain growth proceeds.

\section{Electron back-scatter diffraction (EBSD) and transmission electron microscopic (TEM) analyses}

An extra step of Struers OPS polishing was performed after the standard polishing procedures ${ }^{10}$ to prepare the samples for the EBSD experiments, which were performed on a JEOL JMS-7600F SEM equipped with an Oxford EBSD system. The step sizes used for as-built and deformed samples were $1 \mu \mathrm{m}$ and $0.3 \mu \mathrm{m}$, respectively. Light etching was performed on the as-built samples to review the melt tracks. Post-deformation EBSD graphs were taken $\sim 3 \mathrm{~mm}$ away from the fracture site. Data analysis was performed with the open-source software MTEX ${ }^{27}$. TEM studies were carried on a JOEL TEM 2010 at an accelerating voltage of $200 \mathrm{kV}$. The samples were prepared similarly to those in a previous study ${ }^{10}$.

\section{Results}

\section{Simultaneously enhanced strength and ductility}

The overall SLM processing route is illustrated in Fig. 1. By manipulating the laser power and scanning strategy 


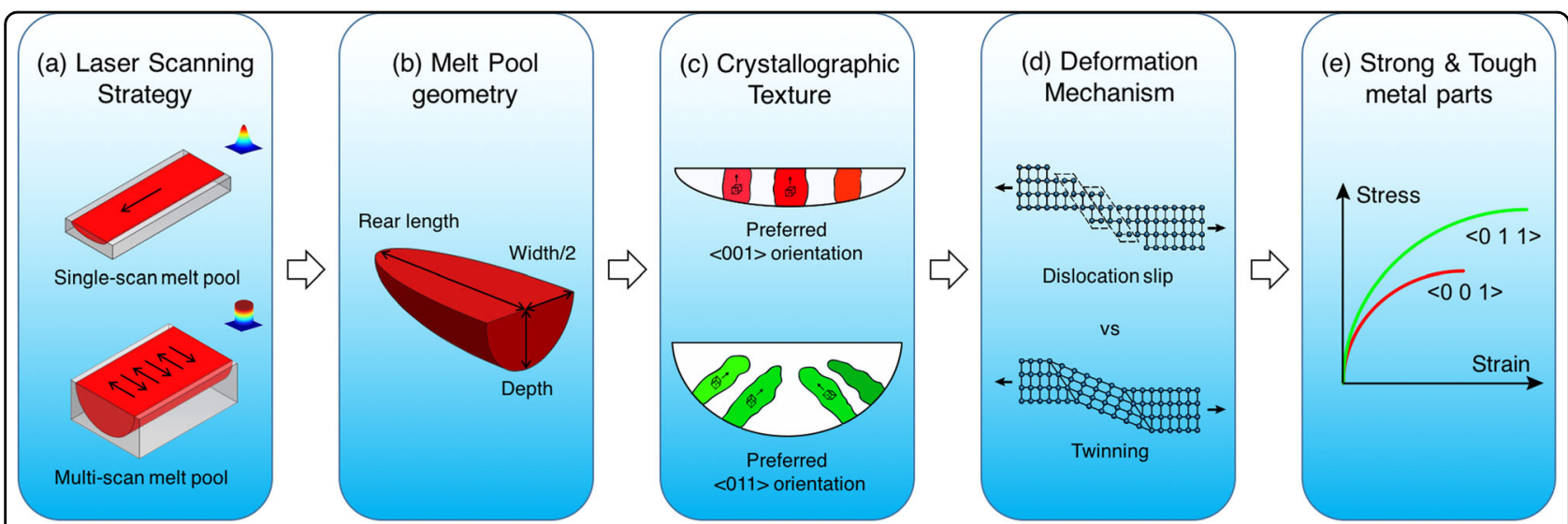

Fig. 1 Overview flowchart of the design of desired properties by means of an SLM AM process. a Laser scanning strategies for single-scan and multi-scan melt pools using different laser process parameters. $\mathbf{b}$ The three key geometrical features (width, depth, and rear length) of a melt pool affecting grain growth. $\mathbf{c}$ Schematic illustration showing the formation of $\langle 001\rangle$ and $\langle 011\rangle$ crystallographic textures due to the different melt pool geometries. $\mathbf{d}$ Schematics of the two prevailing deformation mechanisms (dislocation slip and twinning) in metals and alloys. e The expected outcome of simultaneously enhanced strength and ductility for the SLM-built parts

(Fig. 1a), the geometrical features of the melt pool varied accordingly (Fig. 1b). It would affect the crystallographic textures (Fig. 1c) formed within the built part and the deformation mechanisms (Fig. 1d) active in the deformation process, which could result in different mechanical properties, as shown in Fig. 1e. The SLM process parameters with different power inputs are given in Supplementary Table 1. Samples in this work built with $100 \mathrm{~W}$ power suffered from poor relative densities due to the lack-of-fusion defects caused by insufficient melting. The XRD results of the recycled SS316L powder and the SLM-built SS316L samples are displayed in Fig. 2a. Only FCC $\gamma$-phase austenite was detected in all SLM-built samples. From the XRD peak intensity distribution, we can see that the $100 \mathrm{~W}$ sample shows a similar polycrystalline XRD profile to that of the recycled powder. In contrast, the $200 \mathrm{~W}$ and $380 \mathrm{~W}$ samples have the strongest (200) peak, and the $570 \mathrm{~W}, 760 \mathrm{~W}$ and $950 \mathrm{~W}$ samples possess the strongest (220) peak. This suggests that they may have different crystallographic textures, which will be further elaborated by the EBSD study in the following section.

Figure $2 \mathrm{~b}$ shows the tensile properties of SLM-built SS316L samples with varying process parameters. The mechanical requirements for SS316L based on ASTM A240/A240M ${ }^{28}$ are indicated in Fig. 2b. It is worth noting that samples with similar crystallographic textures had very close tensile performances. Thus only the $100 \mathrm{~W}$, $380 \mathrm{~W}$ and $950 \mathrm{~W}$ samples will be discussed hereafter for clarity. Among all the samples, the $100 \mathrm{~W}$ sample had the lowest tensile strength and elongation values owing to its relatively high porosity. Its strength meets the ASTM standard, but its elongation is poor. We note that the strength of the $380 \mathrm{~W}$ sample far exceeds the ASTM standard. However, its elongation is not satisfactory and is still below the standard. Interestingly, the $950 \mathrm{~W}$ sample exhibits higher strength and better ductility than the 380 W counterpart. The increase in the true ultimate tensile strength and true strain can be up to $\sim 16 \%$ and $\sim 40 \%$, respectively. Similar tensile results along the SLM build direction are shown in Supplementary Fig. 2. Figure 2c shows the work hardening rate curves normalized by a shear modulus of $74 \mathrm{GPa}$ for SS316 ${ }^{29}$. All samples display a general decreasing trend, and the work hardening regimes fall within $0.01-0.03 \mathrm{G}$, which is comparable to other FCC metals with deformation twins ${ }^{30,31}$.

A closer examination of the work hardening rate graphs shows that deformation twinning started earlier for the $950 \mathrm{~W}$ sample at a true strain of $\sim 0.020$ compared to $\sim 0.025$ for the $380 \mathrm{~W}$ sample. These values correspond to the experimental twinning stresses, $\sigma_{\mathrm{tw}}(950 \mathrm{~W})=\sim 585$ $\mathrm{MPa}$ and $\sigma_{\mathrm{tw}}(380 \mathrm{w})=\sim 567 \mathrm{MPa}$, from Fig. 2b. As known, the deformation twinning stress $\sigma_{\mathrm{tw}}$ was derived from $\sigma_{\mathrm{tw}}$ $=2 \times \gamma_{\mathrm{sf}} /\left(b_{\mathrm{p}} \times m_{\text {average }}\right)^{32}$, where $\gamma_{\mathrm{sf}}$ is the stacking fault energy $\left(\sim 20 \mathrm{~mJ} / \mathrm{m}^{2}\right)^{24}, \quad b_{\mathrm{p}}$ is the Burgers vector for Shockley partial dislocation $(\sim 0.146 \mathrm{~nm})$, and $m$ is the Schmid factor. In this work, the area-normalized Schmid factors for the $950 \mathrm{~W}$ and $380 \mathrm{~W}$ samples were determined to be 0.451 and 0.459 , respectively, from their EBSD maps. Therefore, the calculated twining stresses are $\sigma_{\mathrm{tw}(950 \mathrm{~W})}=607 \mathrm{MPa}$ and $\sigma_{\mathrm{tw}(380 \mathrm{~W})}=597 \mathrm{MPa}$, which are in good agreement with the experimental results. Despite its earlier initiation of deformation twinning, the $950 \mathrm{~W}$ sample goes through a longer period of work hardening. This is mainly attributed to the higher quantity of deformation twins in the deformed $950 \mathrm{~W}$ sample, which can be confirmed by the post-deformation EBSD and TEM analyses. 


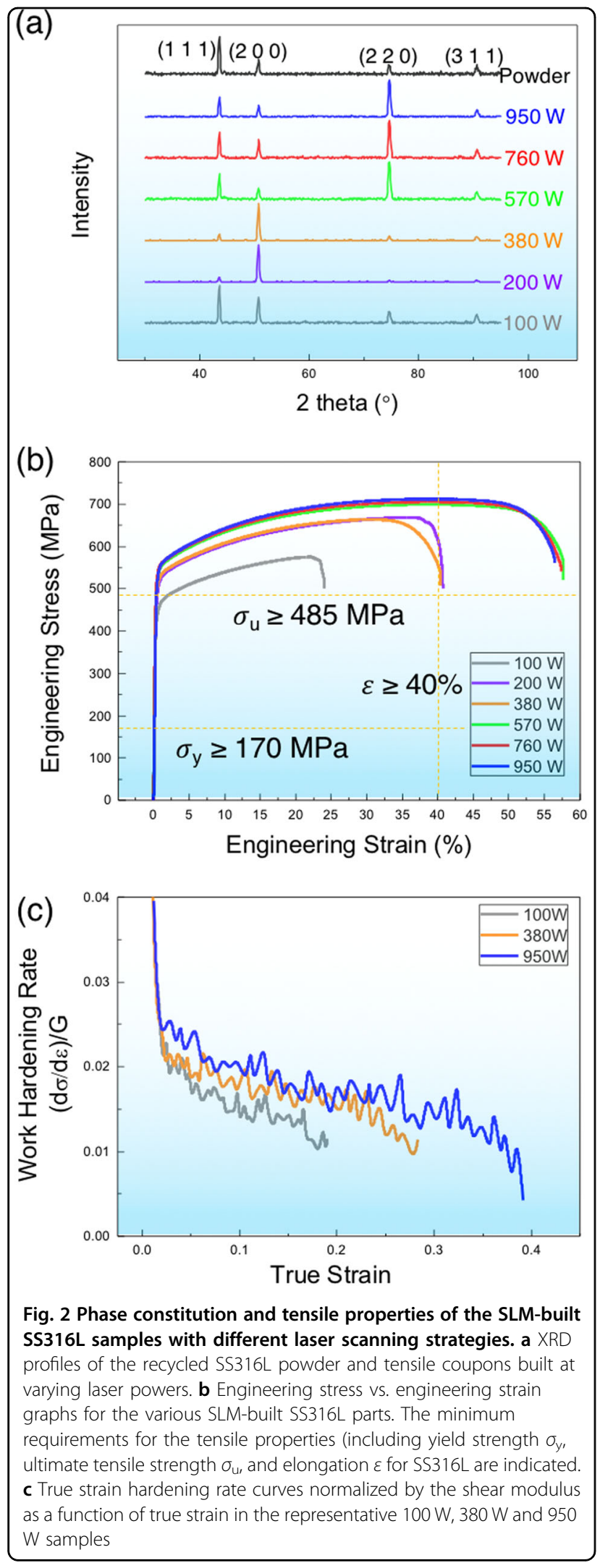

\section{Microstructure and crystallographic texture}

Figure 3 shows that cellular microstructure is present in the $380 \mathrm{~W}$ and $950 \mathrm{~W}$ samples due to the fast cooling rates ${ }^{10}$. With different laser power inputs and laser scanning lengths, however, the size and morphology of the melt pools in the two samples drastically differed. As illustrated in Fig. 1a, the $380 \mathrm{~W}$ sample shows a singlescan melt pool with a Gaussian laser profile, whereas the $950 \mathrm{~W}$ sample shows a multi-scan melt pool with a tophat laser profile. Figure $3 \mathrm{a}$, d show the $X Y$ crosssectional OM images of the $380 \mathrm{~W}$ and $950 \mathrm{~W}$ samples, from which the differences in laser scanning length and hatch spacing can be clearly observed. The $950 \mathrm{~W}$ melt pool is several times larger in size than that of $380 \mathrm{~W}$, as shown in Fig. 3b, e. There exists an obvious epitaxial cellular microstructure in the $380 \mathrm{~W}$ sample, but we only observed an obscure non-epitaxial and misaligned cellular microstructure in the $950 \mathrm{~W}$ sample (Fig. 3c, f). The cellular morphologies arise from similar temperature gradient $\left(G_{\mathrm{L}}\right)$ vs. solidification velocity $\left(V_{\mathrm{L}}\right)$ conditions experienced by both samples ${ }^{33}$. In situ heat treatment effects from the multi-scan melt pool reduce the propensity of elemental segregation, leading to an obscure sub-grain microstructure in the $950 \mathrm{~W}$ sample. The black holes were generated by pitting corrosion during the etching process ${ }^{34}$. The strong Marangoni effect and the high recoil pressure result in strong convection flow within the large $950 \mathrm{~W}$ melt pool, which may induce misaligned cellular growth.

Figure $4 \mathrm{a}, \mathrm{b}$ show the EBSD orientation maps of the 380 $\mathrm{W}$ and $950 \mathrm{~W}$ samples on the $X Z$ plane. The $380 \mathrm{~W}$ sample has a crystallographic texture index of $\sim 3.6$ along the $<001>$ direction, and the $950 \mathrm{~W}$ sample possesses a texture index of $\sim 2.1$ along the $\langle 011\rangle$ direction, as computed from their corresponding inverse pole figures (IPFs), as shown in Fig. 4c, d. Despite the larger melt pool formed in the $950 \mathrm{~W}$ sample, it yields a much smaller average grain area of $\sim 180 \mu \mathrm{m}^{2}$ compared to that of $\sim 310$ $\mu \mathrm{m}^{2}$ for the $380 \mathrm{~W}$ sample. The melt pool boundaries in the $950 \mathrm{~W}$ sample are marked by black solid lines for clarity. By following the heat transfer path, it is clear that all grains were grown perpendicular to the melt pool boundaries. Moreover, fine equiaxed grains were formed on the periphery of the melt pool boundaries. Unlike the epitaxial grain growth along the build direction in the 380 W sample, a "zig-zag", non-epitaxial growth mode is clearly observed in the $950 \mathrm{~W}$ sample. It is noted that the microstructures of both the $380 \mathrm{~W}$ and $950 \mathrm{~W}$ sample remain stable after heat treatment at $650^{\circ} \mathrm{C}$ for $2 \mathrm{~h}$, as shown in Supplementary Fig. 3.

To better understand the relationship between the melt pool geometry and the crystallographic texture, kinetic Monte Carlo simulations were conducted incorporating three key parameters, i.e., width, depth, and rear length of 

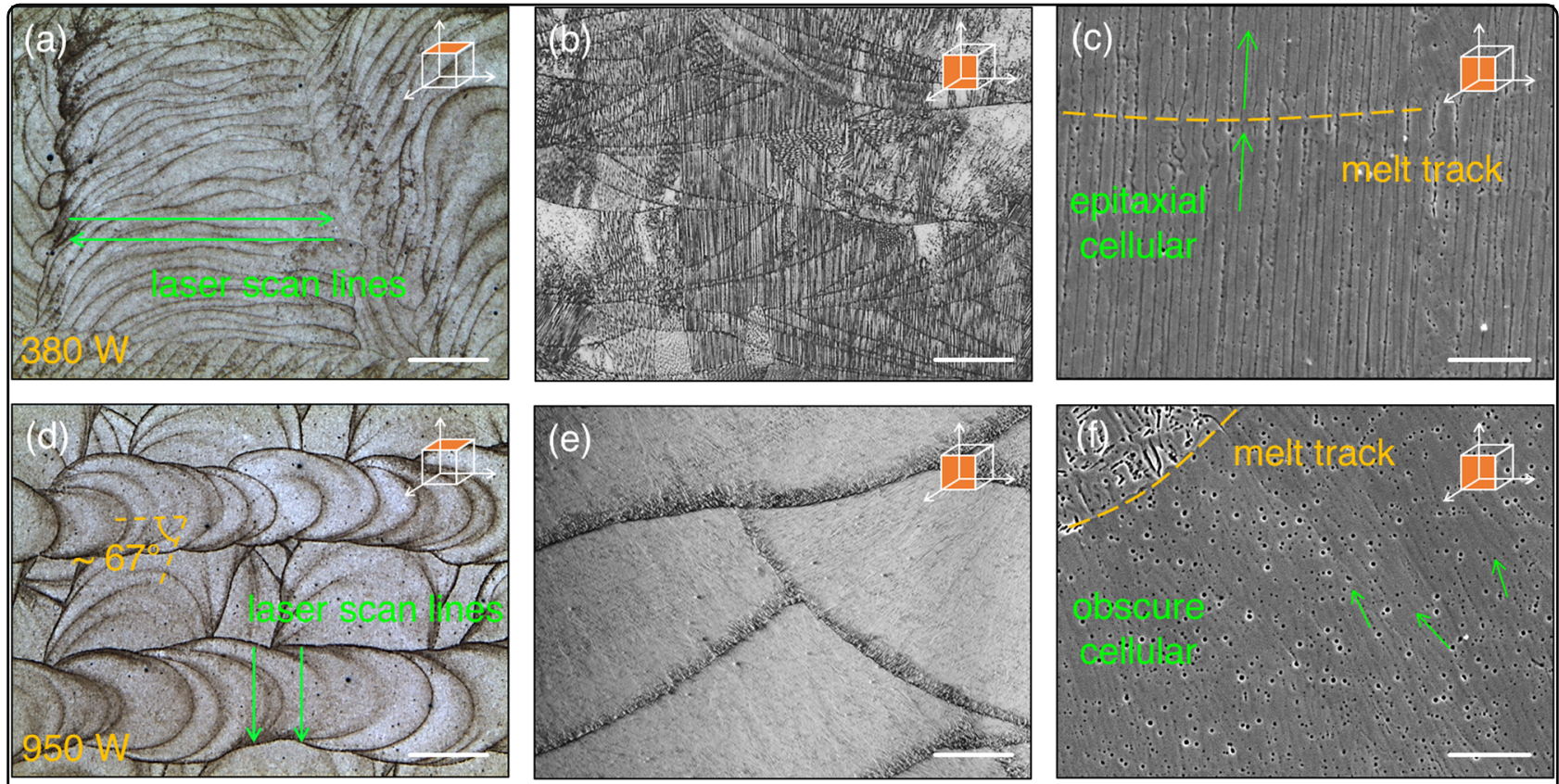

Fig. 3 Melt tracks and microstructures of SLM-built SS316L samples using laser powers of $\mathbf{3 8 0} \mathbf{W}$ and $950 \mathrm{~W}$. Top-and side-view OM images of the $\mathbf{a}, \mathbf{b} 380 \mathrm{~W}$ sample and $\mathbf{d}$, e $950 \mathrm{~W}$ sample. Side-view SEM images of the c $380 \mathrm{~W}$ sample and $\mathbf{f} 950 \mathrm{~W}$ sample. Scale bars for $\mathbf{a}, \mathbf{d}$ are $200 \mu \mathrm{m}, \mathbf{b}$, e are $50 \mu \mathrm{m}$, and $\mathbf{c}, \mathbf{f}$ are $10 \mu \mathrm{m}$

the melt pool geometry, as illustrated in Fig. 1b. Only the rear part of the melt pool was computed, as the front part has little effect on grain growth ${ }^{35}$. Owing to the different melt pool geometries, the grain growth directions are vastly different within each of the melt pools in the two abovementioned samples (see Fig. 1c). Figure 4e, g depict the isometric views of the grain morphologies within the melt pools of the $380 \mathrm{~W}$ and $950 \mathrm{~W}$ samples, respectively. The melt pool widths and depths were taken directly from OM observations (Supplementary Fig. 4). The rear length of the melt pool was captured either from finite element simulations or observation of its ripple pattern. The ratio of the melt pool width, height, and rear length is 6:1:35 and 2:1:3.6 for the $380 \mathrm{~W}$ and $950 \mathrm{~W}$ samples, respectively. It is apparent that grains tend to grow straight from bottom to top within the $380 \mathrm{~W}$ melt pool (Fig. 4f). However, in the $950 \mathrm{~W}$ melt pool, the grains grow perpendicular to the melt pool boundary (Fig. 4h). There is a cluster of equiaxed grains within the center of the melt pool, which prevents the columnar grains from further growth. Moreover, fine equiaxed grains are also present at the periphery of melt pool boundary. Therefore, the experimental observation of the $<011>$ crystallographic texture in the $950 \mathrm{~W}$ sample is well consistent with the simulation results. Videos of the detailed simulation results of these two types of melt pools can be viewed in Supplementary Video 1 and Supplementary Video 2. In addition, the systematic simulation results regarding the influence of the melt pool geometry on the crystallographic texture can be seen in Supplementary Fig. 5.

\section{Influence of laser power and scanning strategy}

It is demonstrated that by employing a specific laser power and scanning strategy, the crystallographic texture and grain size can be engineered through an SLM process. Different tensile behavior may be observed, provided that different deformation mechanisms prevail (see Fig. 1d, e). However, the laser power is not the sole determining factor for the formation of crystallographic textures. It has been reported that a lower laser power of $400 \mathrm{~W}$ produces a $<011>$ texture, whereas a higher power of $1 \mathrm{~kW}$ generates a $<001>$ texture $^{36}$ for the same material (SS316L). Thus a suitable scanning strategy catering to a specific laser power is the key to engineering the crystallographic texture.

Dislocation slip and deformation twinning are the two competing and complementary plastic deformation mechanisms. Deformation twinning is favored because of its superior dislocation storage capacity, which benefits work hardening and ductility enhancement ${ }^{21}$. Figure $5 \mathrm{a}$, c show the $X Y$-plane EBSD graphs of deformed $380 \mathrm{~W}$ and $950 \mathrm{~W}$ samples. Many deformation-induced twins can be observed in certain grains, which are highlighted in the band contrast maps for clarification in Fig. 5b, d. Though nanoscale twins may be absent due to the limited resolution of EBSD scanning $(0.3 \mu \mathrm{m}$ step size), the detected twins in the $380 \mathrm{~W}$ sample are larger but fewer in 

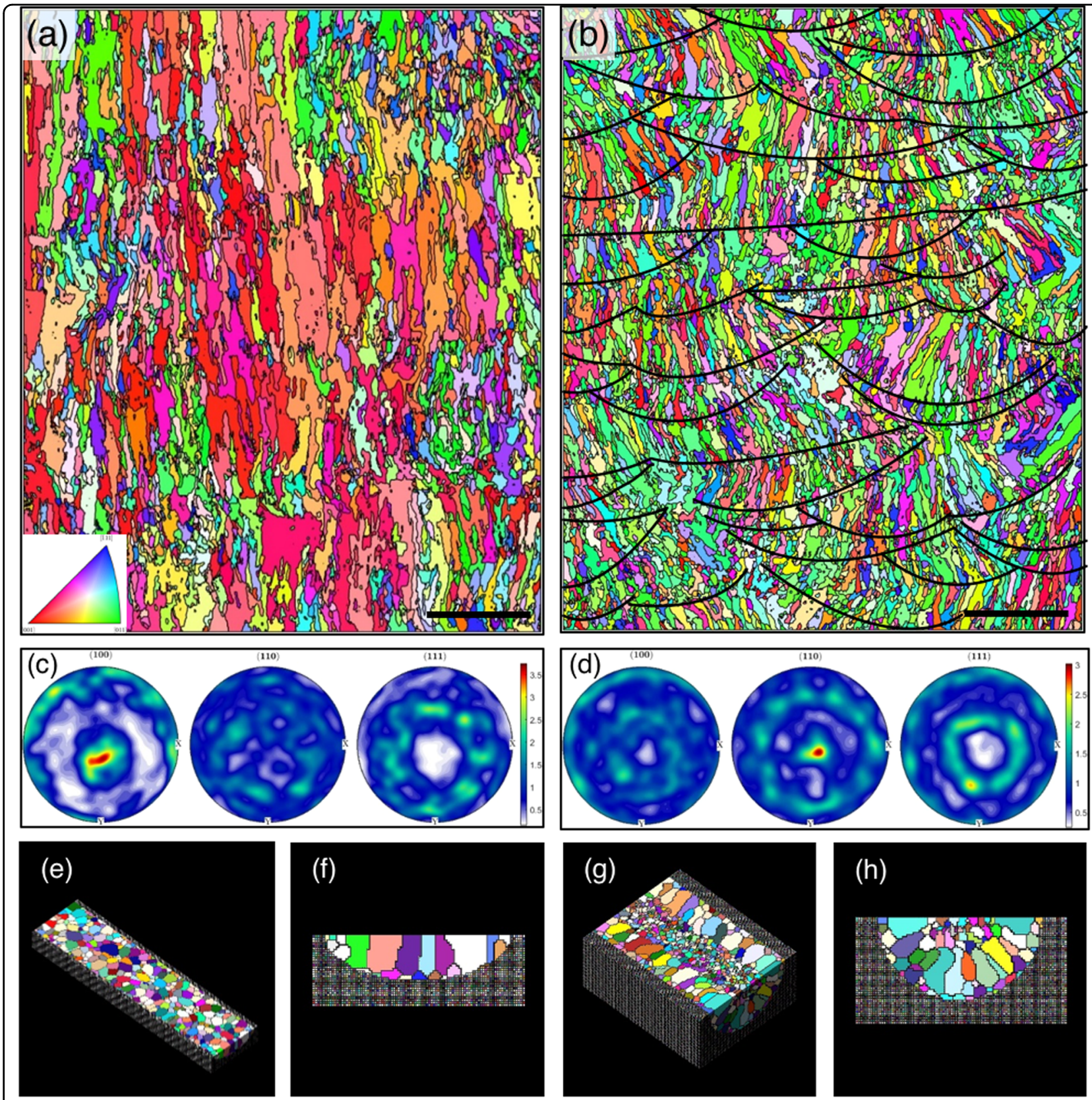

Fig. 4 Formation of $<001>$ and $<011>$ crystallographic textures in SLM-built SS316L samples with laser powers of $380 \mathrm{~W}$ and $950 \mathrm{~W}$, respectively. EBSD IPF color maps with respect to build direction and their respective pole figures for the $\mathbf{a}, \mathbf{c} 380 \mathrm{~W}$ sample and $\mathbf{b}$, d $950 \mathrm{~W}$ sample. Isometric and front views of the kinetic Monte Carlo simulation results for the $\mathbf{e}, \mathbf{f} 380 \mathrm{~W}$ sample and $\mathbf{g}, \mathbf{h} 950 \mathrm{~W}$ sample. Scale bars for $\mathbf{a}, \mathbf{b}$ are $200 \mu \mathrm{m}$

number compared to the $950 \mathrm{~W}$ sample. A total area of $\sim 0.6 \mathrm{~mm}^{2}$ was examined by EBSD for the deformed $380 \mathrm{~W}$ and $950 \mathrm{~W}$ samples. The overall detected twin length in the deformed $950 \mathrm{~W}$ sample $(\sim 17.0 \mathrm{~mm})$ is $\sim 25 \%$ longer than that of the deformed $380 \mathrm{~W}$ sample $(\sim 13.6 \mathrm{~mm})$.

Figure 5e, g show the corresponding IPFs of Fig. 5a, c along the tensile direction. Grains with and without deformation twins are marked by red and black dots. The dotted contour lines indicate the Taylor factor $(M)$. It is apparent that deformation-induced twinning hardly appears when $M<2.6$ and the majority of twinned grains are aligned close to the $<111>$ orientation along the tensile direction. This is consistent with previous findings from other types of austenitic steels ${ }^{37-39}$. It is accepted that the activation of multiple slip systems and the presence of dislocation pile-ups are necessary to induce twinning during deformation ${ }^{40}$. Grains with a higher 

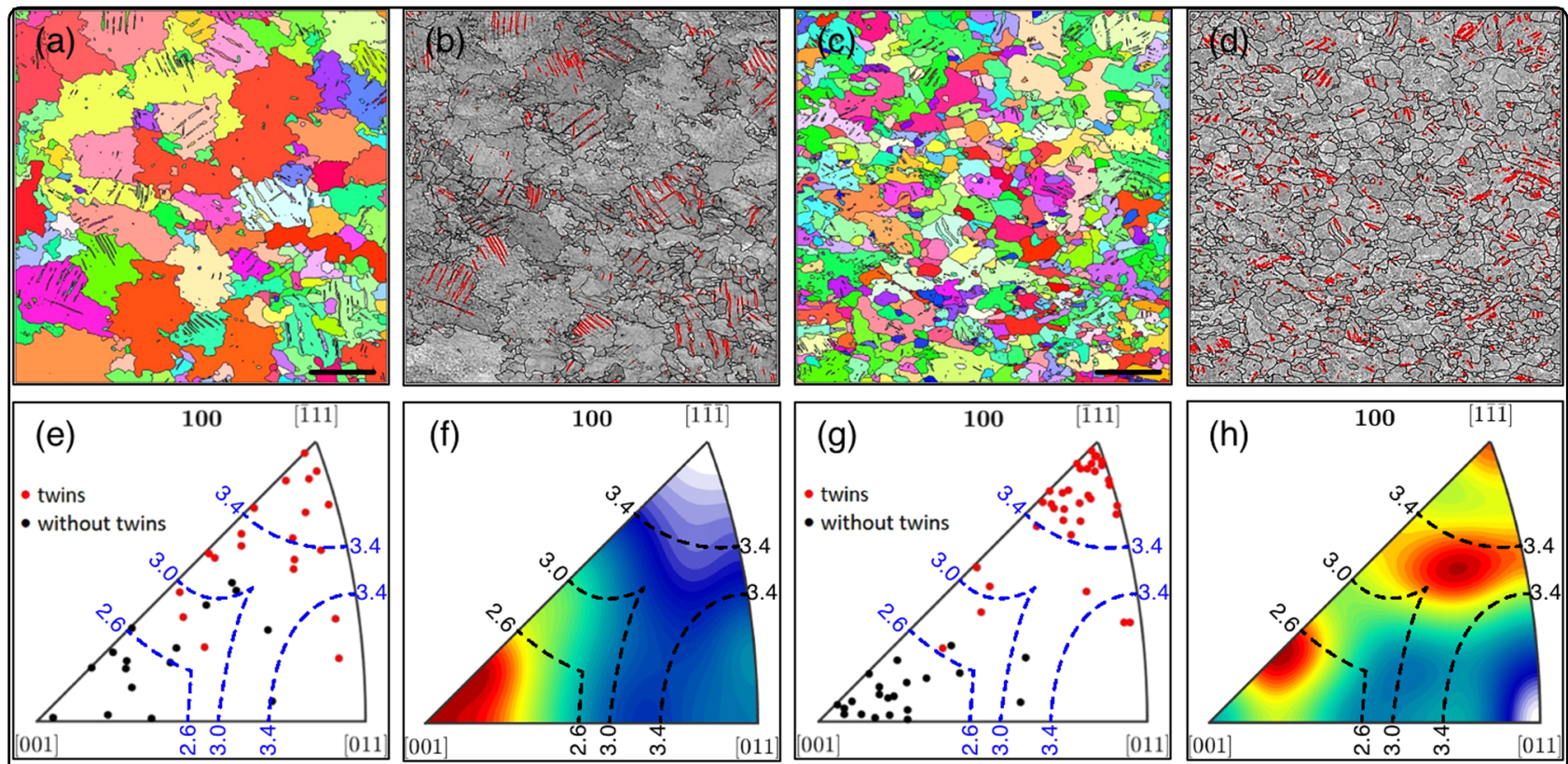

Fig. 5 Occurrence of deformation twins in SLM-built SS316L samples after plastic deformation. EBSD IPF color maps for the top view of deformed $\mathbf{a} 380 \mathrm{~W}$ and $\mathbf{c} 950 \mathrm{~W}$ samples. The respective band contrast maps superimposed with red-colored deformation twins are shown in $\mathbf{b}$ and d. The inverse pole figures consisting of grains with and without deformation twins parallel to the tensile direction are shown in $\mathbf{e}$ and $\mathbf{g}$. The inverse pole figures of the as-built $\mathbf{f} 380 \mathrm{~W}$ and $\mathbf{h} 950 \mathrm{~W}$ samples parallel to the tensile direction. The dotted contour lines represent the Taylor factors. Scale bars for $\mathbf{a}, \mathbf{c}$ are $50 \mu \mathrm{m}$

Taylor factor $(M>2.6)$ experienced a greater amount of crystallographic shear with regards to the uniaxial tension, which in turn resulted in additional strain hardening and a higher dislocation density to maintain strain compatibility with the adjoining grains ${ }^{41}$. Moreover, it is revealed that grains with a lower Taylor factor $(M<2.6)$ may not have sufficient slip systems and/or dislocations to induce deformation twinning. Figure $5 \mathrm{f}$, $\mathrm{h}$ show the IPFs from the two EBSD maps of the as-built $380 \mathrm{~W}$ and $950 \mathrm{~W}$ samples in Fig. 4. It can be derived from Fig. $5 \mathrm{f}$ that most of the grains in the $380 \mathrm{~W}$ sample were unfavorable to induce deformation twinning, at least at the initial deformation stage, due to the strong $<001>$ crystallographic texture and low value of $M(<2.6)$ along the tensile direction. In contrast, due to the $<011>$ crystallographic texture formed in the 950 $\mathrm{W}$ sample, the grains were not entirely constrained within the region of $M<2.6$. Thus a larger number of deformation twins could be formed during the plastic deformation process. Moreover, as suggested by recent relevant studies, residual stress does not seem to play a significant role in the deformation process for SLM-processed SS316L ${ }^{42-44}$. Thus the influence of residual stress on the mechanical deformation behavior may not be a main concern in this work.

TEM study of the as-SLM-built SS316L samples reveals that more dislocations exist in the $950 \mathrm{~W}$ sample than in the $380 \mathrm{~W}$ sample (see Supplementary Fig. 6). Figure 6a shows two adjacent grains with two different orientations $(<001>$ and $<321>)$ in the deformed $380 \mathrm{~W}$ sample. No twins were observed within the $<001>$-oriented grain. The cubic texture of (001)[100] developed in the $380 \mathrm{~W}$ sample further confirms that grains with an orientation close to $<001>$ alignment along the tensile direction are unfavorable for deformation twinning. However, within the non- $<001>$-oriented grains, separated single-twin lamellae with tens of nanometers in width are commonly seen in the $380 \mathrm{~W}$ sample (Fig. 6a-c). Moreover, Fig. 6b, c display a large number of dislocations and stacking faults between the twin lamellae, which likely strongly interact with each other during plastic deformation. In contrast, many more multiple deformation twin lamellae were observed in the deformed $950 \mathrm{~W}$ sample (see Fig. 6d). The multiple nano-twin lamellae and the stacking fault regions are shown in Fig. 6e. The highresolution transmission electron microscopic image in Fig. $6 \mathrm{f}$ reveals the nano-twin lamellae and stacking faults on the atomic scale. Stacking faults composed of partial dislocations were formed owing to the dislocation/twin interactions ${ }^{19}$. It is evidenced that twinning is the dominant deformation mechanism in the $<011>$-textured 950 $\mathrm{W}$ sample and the formation of nano-twins could be the major reason for the simultaneously enhanced strength and ductility.

\section{Discussion}

The present study reveals that the strength and ductility of SS316L can be simultaneously enhanced via an SLM 

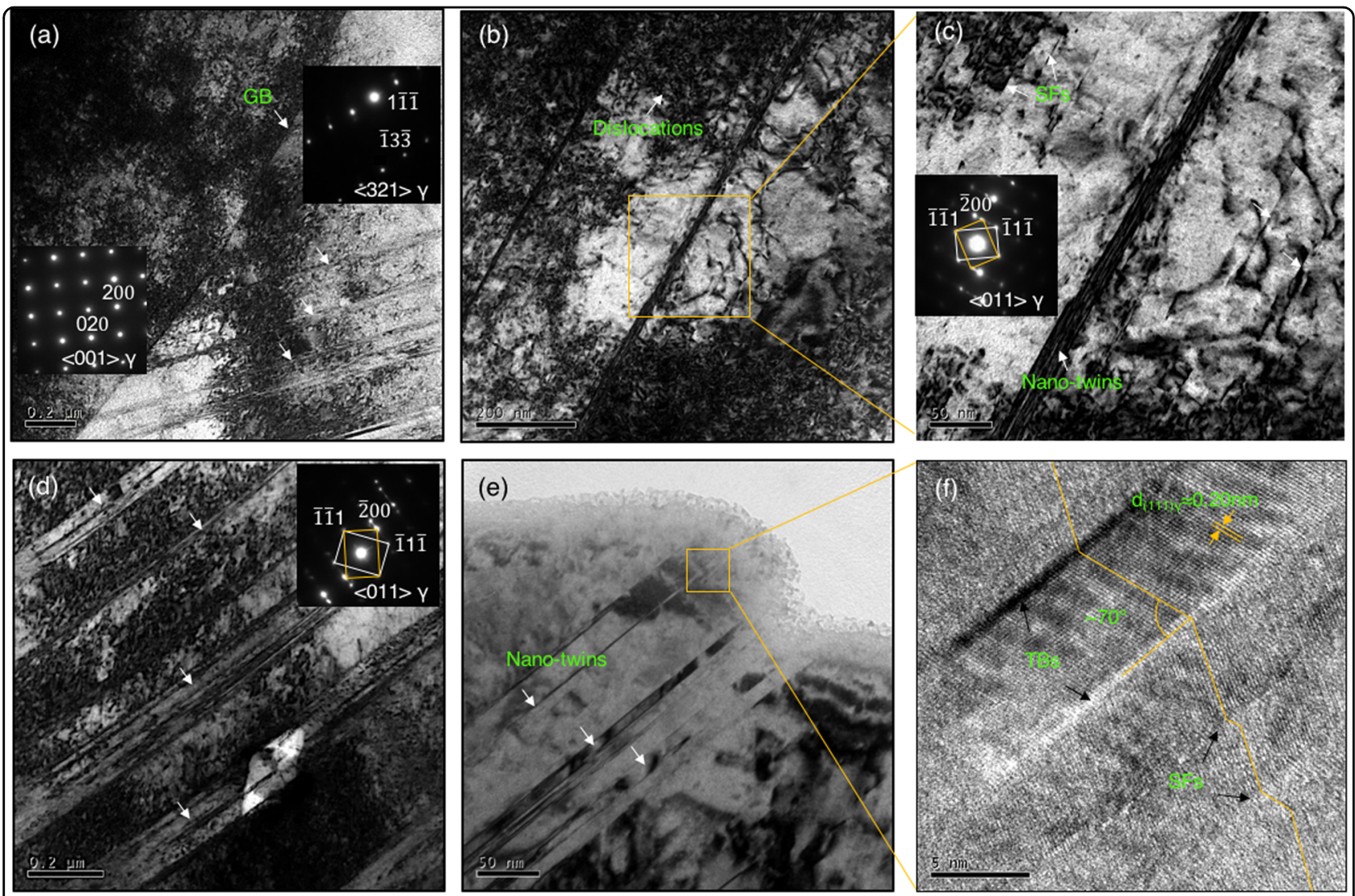

Fig. 6 TEM micrographs showing the dominant deformation mechanisms in the two representative SLM-built SS316L samples with different crystallographic textures. a TEM image showing two adjacent grains with $<001>$ and $<321>$ orientations in the deformed $380 \mathrm{~W}$ sample. The grain boundary (GB) is indicated. The right three arrows point to the single-twin lamellae. $\mathbf{b}$ TEM image showing dislocations and stacking faults between two single-twin lamellae in the deformed $380 \mathrm{~W}$ sample. $\mathbf{c}$ Enlarged TEM image of the enclosed region in $\mathbf{b}$ showing a nano-twin and surrounding stacking faults (SFs) along the $\langle 011>$ zone axis. $\mathbf{d}$ TEM image showing a large number of multiple twins indicated by arrows in the deformed $950 \mathrm{~W}$ sample along the $<011>$ zone axis. e TEM image showing the multiple nano-twins in the deformed $950 \mathrm{~W}$ sample. f HRTEM image of the enclosed region in e showing the nano-twin lamellae with twin boundaries (TBs) and SFs. Scale bars for $\mathbf{a}, \mathbf{b}, \mathbf{d}$ are $200 \mathrm{~nm}$, for $\mathbf{c}, \mathbf{e} 50 \mathrm{~nm}$, and for $\mathbf{f} 5 \mathrm{~nm}$

process by tailoring a specific crystallographic texture. It was shown that the melt pool geometry has a significant influence on the formation of the crystallographic texture. From the previous studies on single laser tracks, we know that slow scanning generates deep but irregular melt pools, while fast scanning creates discontinuous balling phenomena ${ }^{35,45}$. Therefore, the majority of the optimized laser scanning parameters fall in between, producing regular, shallow melt pools. This in turn contributes to the formation of a $<001>$ crystallographic texture along the build direction in SLM-built metal parts. In this work, we propose a multi-scan melt pool strategy (Fig. 1a) that can produce a stable, deep melt pool with a width-toheight ratio of $2: 1$ by utilizing a high-power $(>400 \mathrm{~W})$ laser with a top-hat profile. Accordingly, a desirable $<011>$ crystallographic texture is formed instead of the common <001> crystallographic texture. Utilizing the kinetic Monte Carlo mesoscale simulation approach, it is shown that a long, shallow melt pool promotes the formation of $<001\rangle$ texture, whereas a short, deep melt pool favors the occurrence of $\langle 011>$ texture (see Supplementary Fig. 5). Obviously, both the experimental and simulation results demonstrate that we can effectively engineer the crystallographic textures within the SLM-processed metal parts by adjusting the melt pool geometry via different laser beam process parameters. Of particular note is that the crystallographic texture selection map shown in Supplementary Fig. 5 can also be applied to other powderbased AM processes, such as electron beam melting and laser engineered net shaping. This work provides meaningful guidance for the fabrication of complex metal parts with desired properties via microstructural engineering in a beam-based, layer-wise material deposition process.

Deformation twinning is unfavorable in the <001> orientation along the tensile direction ${ }^{35}$. However, most metal AM parts, such as the $380 \mathrm{~W}$ sample in this work, 
have a $<001>$ crystallographic texture. Though grain rotations may occur due to intragranular compatibility during the elongation process, the deformation twinning capacity is greatly limited. In contrast, the $<011>$ crystallographic texture that formed in the $950 \mathrm{~W}$ sample enables the activation of more deformation twins within the grains, particularly for the formation of nano-twins, allowing higher strains. The IPF results showed that this is the case for directions both perpendicular and parallel to the build direction. Moreover, the smaller grain size and higher amount of initial dislocation density in the $950 \mathrm{~W}$ sample contribute to a higher yield strength. Therefore, a higher yield strength accompanied by an earlier initiation of deformation twinning and a longer strain hardening regime due to the specific $<011>$ crystallographic texture makes the high-power SLM-built samples stronger and tougher.

The findings of the present work will be of significance to additively manufacture other types of metallic materials into high-performance parts, especially those with a low stacking fault energy. This work also helps explain and predict the formation of certain crystallographic textures during rapid solidification processes, such as welding and laser surface modification. Engineering crystallographic textures in the microstructure via an SLM process is a promising method to overcome the long-standing strength-ductility dilemma in metallic engineering materials. With enhanced strength and ductility, metal AM parts or components will become safer when used in heavy load-bearing structures, and less material will be needed to achieve the same goal. Moreover, the use of a high-power laser can effectively improve the AM fabrication rate. These findings can help metal AM find broader industrial application.

\section{Acknowledgements}

The authors acknowledge the use of the resources of the Singapore Centre for 3D Printing funded by the National Research Foundation (NRF), Singapore.

\section{Authors' contributions}

X.T. conceived the idea and organized the data. Z.S. carried out the SLM fabrications, microstructural and mechanical characterization, and MC simulations. X.T. helped with the TEM observation. Z.S. and X.T. wrote the manuscript. All authors discussed the results and commented on the manuscript.

\section{Conflict of interest}

The authors declare that they have no conflict of interest.

\section{Publisher's note}

Springer Nature remains neutral with regard to jurisdictional claims in published maps and institutional affiliations.

Supplementary information is available for this paper at https://doi.org/ 10.1038/s41427-018-0018-5.

Received: 12 October 2017 Revised: 6 January 2018 Accepted: 8 January 2018.

Published online: 10 April 2018

\section{References}

1. Martin, J. H. et al. 3D printing of high-strength aluminium alloys. Nature $\mathbf{5 4 9}$, 365-369 (2017).

2. Tan, $X$. et al. Revealing martensitic transformation and $\alpha / \beta$ interface evolution in electron beam three-dimensional-printed Ti-6Al-4V. Sci. Rep. 6 , 26039 (2016).

3. Geiger, F., Kunze, K. \& Etter, T. Tailoring the texture of IN738LC processed by selective laser melting (SLM) by specific scanning strategies. Mater. Sci. Eng. A 661, 240-246 (2016).

4. Frazier, W. E. Metal additive manufacturing: a review. J. Mater. Eng. Perform. 23, 1917-1928 (2014).

5. Tan, X. et al. Graded microsctructure and mechanical properties of additive manufactured Ti-6Al-4V via electron beam melting. Acta Mater. 97, 1-16 (2015).

6. Vrancken, B., Thijs, L., Kruth, J.P. \& Van Humbeeck, J. Heat treatment of Ti6Al4V produced by selective laser melting: microstructure and mechanical properties. J. Alloy. Compd. 541, 177-185 (2012).

7. Lam, L. P. et al. Phase analysis and microstructure characterisation of AlSi10Mg parts produced by selective laser melting. Virtual Phys. Prototyp. 10, 207-215 (2015).

8. $\mathrm{Xu}, \mathrm{W}$. et al. Additive manufacturing of strong and ductile Ti- $6 \mathrm{Al}-4 \mathrm{~V}$ by selective laser melting via in situ martensite decomposition. Acta Mater. 85, 74-84 (2015).

9. Mumtaz, K. A., Erasenthiran, P. \& Hopkinson, N. High density selective laser melting of Waspaloy ${ }^{\circledast}$. J. Mater. Process. Technol. 195, 77-87 (2008).

10. Kunze, K., Etter, T., Grässlin, J. \& Shklover, V. Texture, anisotropy in microstructure and mechanical properties of IN738LC alloy processed by selective laser melting (SLM). Mater. Sci. Eng. A 620, 213-222 (2015).

11. Sun, Z., Tan, X., Tor, S. B. \& Yeong, W. Y. Selective laser melting of stainless steel 316L with low porosity and high build rates. Mater. Des. 104, 197-204 (2016).

12. Ishimoto, T., Hagihara, K., Hisamoto, K., Sun, S.-H. \& Nakano, T. Crystallographic texture control of beta-type Ti-15Mo-5Zr-3Al alloy by selective laser melting for the development of novel implants with a biocompatible low Young's modulus. Scr. Mater. 132, 34-38 (2017).

13. Haase, C. et al. Exploiting process-related advantages of selective laser melting for the production of high-manganese steel. Materials 10, 56 (2017).

14. Zhou, $X$. et al. Textures formed in a CoCrMo alloy by selective laser melting. J. Alloy. Compd. 631, 153-164 (2015).

15. Thijs, L. et al. Strong morphological and crystallographic texture and resulting yield strength anisotropy in selective laser melted tantalum. Acta Mater. 61, 4657-4668 (2013).

16. Carter, L. N., Martin, C., Withers, P. J. \& Attallah, M. M. The influence of the lase scan strategy on grain structure and cracking behaviour in SLM powder-bed fabricated nickel superalloy. J. Alloy. Compd. 615, 338-347 (2014).

17. Thijs, L., Verhaeghe, F., Craeghs, T., Humbeeck, J. V. \& Kruth, J.P. A study of the microstructural evolution during selective laser melting of Ti-6Al-4V. Acta Mater. 58, 3303-3312 (2010).

18. $\mathrm{Yu}, \mathrm{Q}$. et al. Strong crystal size effect on deformation twinning. Nature 463, 335-338 (2010)

19. Wei, Y. et al. Evading the strength-ductility trade-off dilemma in steel through gradient hierarchical nanotwins. Nat. Commun. 5, 3580 (2014).

20. Li, Z., Pradeep, K. G., Deng, Y., Raabe, D. \& Tasan, C. C. Metastable high-entropy dual-phase alloys overcome the strength-ductility trade-off. Nature 534, 227-230 (2016)

21. Lu, K., Lu, L. \& Suresh, S. Strengthening materials by engineering coherent internal boundaries at the nanoscale. Science 324, 349-352 (2009).

22. Riemer, A. et al. On the fatigue crack growth behavior in $316 \mathrm{~L}$ stainless steel manufactured by selective laser melting. Eng. Fract. Mech. 120, 15-25 (2014).

23. Yan, C., Hao, L., Hussein, A., Young, P. \& Raymont, D. Advanced lightweight $316 \mathrm{~L}$ stainless steel cellular lattice structures fabricated via selective laser melting. Mater. Des. 55, 533-541 (2014).

24. Obrtlík, K., Kruml, T. \& Polák, J. Dislocation structures in $316 \mathrm{~L}$ stainless steel cycled with plastic strain amplitudes over a wide interval. Mater. Sci. Eng. A 187, 1-9 (1994)

25. Plimpton, S., et al. Crossing the Mesoscale No-Man's Land via Parallel kinetic Monte Carlo. Sandia Report SAND2009-6226, Sandia National Laboratories, Albuquerque, NM 87185-1316, (2009). 
26. Rodgers, T. M., Madison, J. D., Tikare, V. \& Maguire, M. C. Predicting mesoscale microstructural evolution in electron beam welding. JOM 68, 1419-1426 (2016).

27. Bachmann, F., Hielscher, R. \& Schaeben, H. Texture Analysis with MTEX - Free and Open Source Software Toolbox., in Solid State Phenomena, edited by Klein, A. \& Schwarzer, R.A. Trans. Tech. Publ., Switzerland, Vol. 160, pp. 63-68 (2010).

28. ASTM A240/A240M-16a. Standard Specification for Chromium and Chromiumnickel Stainless Steel Plate, Sheet, and Strip for Pressure Vessels and for General Applications. ASTM International, West Conshohocken, PA, 2016. (http://www. astm.org).

29. Ya, M., Xing, Y., Dai, F., Lu, K. \& Lu, J. Study of residual stress in surface nanostructured AISI 316L stainless steel using two mechanical methods. Surf. Coat. Technol. 168, 148-155 (2003).

30. Laplanche, G., Kostka, A., Horst, O. M., Eggeler, G. \& George, E. P. Microstructure evolution and critical stress for twinning in the CrMnFeCoNi high-entropy alloy. Acta Mater. 118, 152-163 (2016).

31. El-Danaf, E., Kalidindi, S. R. \& Doherty, R. D. Influence of grain size and stackingfault energy on deformation twinning in fcc metals. Metall. Mater. Trans. A 30, 1223-1233 (1999)

32. Byun, T. S. On the stress dependence of partial dislocation separation and deformation microstructure in austenitic stainless steels. Acta Mater. $\mathbf{5 1}$ 3063-3071 (2003).

33. Rosa, D. M., Spinelli, J. E., Ferreira, I. L. \& Garcia, A. Cellular/dendritic transition and microstructure evolution during transient directional solidification of $\mathrm{Pb}$ Sb alloys. Metall. Mater. Trans. A 39, 2161-2174 (2008).

34. Zhang, K., Zou, J., Grosdidier, T., Dong, C. \& Yang, D. Improved pitting corrosion resistance of AISI $316 \mathrm{~L}$ stainless steel treated by high current pulsed electron beam. Surf. Coat. Technol. 201, 1393-1400 (2006).
35. Liu, W. \& DuPont, J. N. Effects of melt-pool geometry on crystal growth and microstructure development in laser surface-melted superalloy single crystals. Acta Mater. 52, 4833-4847 (2004).

36. Niendorf, T. et al. Highly anisotropic steel processed by selective laser melting. Metall. Mater. Trans. B 44, 794-796 (2013).

37. Gutierrez-Urrutia, I., Zaefferer, S. \& Raabe, D. The effect of grain size and grain orientation on deformation twinning in a Fe-22wt.\% Mn-0.6wt.\% C TWIP steel. Mater. Sci. Eng. A 527, 3552-3560 (2010).

38. Beladi, $\mathrm{H}$. et al. Orientation dependence of twinning and strain hardening behaviour of a high manganese twinning induced plasticity steel with polycrystalline structure. Acta Mater. 59, 7787-7799 (2011).

39. Yang, P., Xie, Q., Meng, L., Ding, H. \& Tang, Z. Dependence of deformation twinning on grain orientation in a high manganese steel. Scr. Mater. 55 629-631 (2006).

40. Karaman, I., Sehitoglu, H., Gall, K., Chumlyakov, Y. I. \& Maier, H. Deformation of single crystal Hadfield steel by twinning and slip. Acta Mater. 48, 1345-1359 (2000).

41. Christian, J. W. \& Mahajan, S. Deformation twinning. Prog. Mater. Sci. 39, 1-157 (1995).

42. Wang, $Y$., et al. Additively manufactured hierarchical stainless steels with high strength and ductility. Nat. Mater. 17, 63-71 (2017).

43. Liu, L., et al. Dislocation network in additive manufactured steel breaks strength-ductility trade-off. Mater. Today, in press (2018).

44. Salmi, A. \& Atzeni, E. History of residual stresses durin gthe production phases of Alsi10Mg parts processed by powder bed additive manufacturing technology. Virtual Phys. Prototyp. 12, 153-160 (2017).

45. Yadroitsev, I., Gusarov, A., Yadroitsava, I. \& Smurov, I. Single track formation in selective laser melting of metal powders. J. Mater. Process. Technol. 210 1624-1631 (2010). 